\title{
Fertile heteroallelic combinations of mutant alleles of the otu locus of Drosophila melanogaster
}

Patrick Daniel Storto and Robert Charles King

Department of Evolutionary Biology, Northwestern University, Evanston, IL 60201, USA

Roux's Arch Dev Biol (1987) 196:210-221

The first complete paragraph in the left-hand column on page 212 should have read:

The matrix in Table 5 displays 72 heteroallelic combinations involving QUI/ONC and QUI/DIF alleles. Data for the eight QUI homozygotes appear in the left column. The matrix in Table 6 displays the 36 heteroallelic combinations involving $\mathrm{ONC}$ and DIF alleles. The diagonal row of nine gives the data for homozygotes. 